\title{
Fluorescein Chemiluminescent Assay of Glucose in Serum Using Glucose Oxidase and Horseradish Peroxidase
}

\author{
Tadashi Segawa, Asako Kakizaki, Tamio Kamidate and Hiroto Watanabe \\ Faculty of Engineering, Hokkaido University, Sapporo 060, Japan
}

\begin{abstract}
Glucose in serum was successfully determined by the simultaneous coupling of a glucose-glucose oxidase (GOD) reaction with a fluorescein (FL)-chemiluminescent (CL) reaction catalyzed by horseradish peroxidase. The FL-CL reaction could be conducted at $\mathrm{pH} 7.0$, thus allowing the in situ detection of hydrogen peroxide generated during the glucose-GOD reaction. The $\mathrm{CL}$ emission lasted for about $3 \mathrm{~min}$, and the calibration curve was linear over a glucose concentration range of $5.0 \times 10^{-8}$ to $1.0 \times 10^{-5} \mathrm{M}$. The relative standard deviation at $5.0 \times 10^{-7} \mathrm{M}$ glucose was $1.8 \%(n=5)$. The present method was not subject to any interference from components normally present in serum. The results of glucose assays in two control sera were in good agreement with those conducted by the colorimetric method.
\end{abstract}

Keywords Chemiluminescence, glucose, fluorescein, peroxidase

Glucose in blood is clinically important in the diagnosis of diabetes or endocrinopathy. In general, glucose is indirectly assayed by measuring the amount of hydrogen peroxide generated during the enzymatic reaction of glucose with glucose oxidase (EC 1.1.3.4; GOD). The peroxide, thus formed, is determined by the use of an appropriate chromogenic or chemiluminescent (CL) reagent. ${ }^{1-10}$ The coupled reaction sequence is expressed by the following equations:

$$
\text { Glucose }+\mathrm{O}_{2} \stackrel{\text { GOD }}{\longrightarrow} \text { gluconic acid }+\mathrm{H}_{2} \mathrm{O}_{2}
$$

and

$$
\mathrm{H}_{2} \mathrm{O}_{2}+\text { reagent } \rightarrow \text { dye formation or } \mathrm{CL} \text { emission. (ii) }
$$

Luminol $^{3,4}$ and peroxyoxalate ${ }^{6,7} \mathrm{CL}$ methods have been employed for the detection of hydrogen peroxide formed during the enzymatic reaction. Inconveniences in the use of these $\mathrm{CL}$ reagents are that the high $\mathrm{pH}$ conditions in the former and the aqueous-organic solvents in the latter are required for efficient $\mathrm{CL}$ emission. This is not compatible with enzymatic reaction (i), which proceeds favorably under mild $\mathrm{pH}$ conditions, thus necessitating the use of a two-stage reaction sequence. Namely, after oxidation of glucose by GOD in a mild pH medium, an alkaline solution or an organic solvent must be added to the medium to initiate the CL reaction.

To eliminate this inconvenience, Igarashi and Hinze have proposed the use of an inverted cationic micellar medium, thus allowing one to perform both enzymatic and luminol-CL reactions simultaneously at mild $\mathrm{pH} .^{5}$ The micellar medium was also successful in conducting the two reactions simultaneously at mild $\mathrm{pH}$, when a peroxyoxalate-fluorescent dye CL system was employed. 9

We previously proposed fluorescein (FL) as a novel, sensitive $C L$ reagent for detecting trace hydrogen peroxide in the presence of horseradish peroxidase (EC 1.11.1.7; HRP). ${ }^{11}$ Subsequently, we elucidated the mechanism of light emission in the FL CL reaction. ${ }^{12}$ The FL CL reaction takes place in an aqueous medium at pH 7 without any modification of the medium with surfactants or organic solvents. In this respect, FL seems to be a promising CL reagent for the analysis of glucose by an in situ coupling of the two reactions. In this work we explored the possibility of applying the FL $\mathrm{CL}$ reaction to the determination of glucose in serum.

\section{Experimental}

\section{Apparatus}

All of the CL measurements were made by using a CL detector TD-3A (Tohoku Densi Sangyo Co., Ltd.) equipped with an automatic injector (Model 500, Nichiryo Co., Ltd.).

\section{Reagents}

The following reagents were used as received: horseradish peroxidase (Sigma, type VI), GOD (Nagase, from Penicillum amagasakiense, not containing catalase), Dglucose (Kanto Chemical), bovine serum alubumin (Wako), and control serum (Wako; Control Serum I and Ortho Diagnostic Systems; Ortho Liquid Normal). All of the aqueous solutions were prepared with water purified using a Millipore Milli Q-II system. 


\section{Procedure}

A $0.50 \mathrm{~cm}^{3}$ portion of a glucose solution was introduced into a CL cuvette $(22 \mathrm{~mm}$ i.d. $\times 20 \mathrm{~mm})$, which was subsequently placed in the holder of the CL counter. A $0.50 \mathrm{~cm}^{3}$ portion of a $\mathrm{CL}$ reagent solution containing $5.0 \times 10^{-8} \mathrm{M}\left(1 \mathrm{M}=1 \mathrm{~mol} \mathrm{dm}^{-3}\right) \mathrm{HRP}, 6.6 \times 10^{-4} \mathrm{M} \mathrm{FL}$ and $4.0 \mathrm{U} \mathrm{cm}^{-3}$ GOD in $0.10 \mathrm{M} \mathrm{3}-(N$-morpholino $)$ propanesulfonic acid (MOPS)-NaOH buffer ( $\mathrm{pH} 7.0$ ) was then added to the cuvette using the injector. The CL reaction was thus initiated, and the CL emission was monitored as a function of time with a photomultiplier tube. The resultant photocurrent was converted to a voltage, the value of which was displayed on a chart recorder. Simultaneously, the emitted photons were counted and integrated for $3 \mathrm{~min}$ from the initiation of CL emission. Unless otherwise stated, the CL intensity was defined as being the number of photons emitted for $3 \mathrm{~min}$, after correcting for the intensity of a blank.

\section{Results and Discussion}

\section{Two-stage reaction sequence and simultaneous reaction} sequence

Since the FL CL reaction proceeds in neutral aqueous solutions, no problems arise due to the use of the FL CL reaction in the two-stage sequence mode. We first tested the two-stage and simultaneous reaction sequences in order to determine which has a higher sensitivity to glucose.

In testing the two-stage reaction sequence, the following procedure was employed. A $10.0 \mathrm{~cm}^{3}$ portion of a $2.0 \times 10^{-5} \mathrm{M}$ glucose solution and a $10.0 \mathrm{~cm}^{3}$ portion of a $8.0 \mathrm{U} \mathrm{cm}^{-3} \mathrm{GOD}$ solution were mixed; and the mixture was then kept for $30 \mathrm{~min}$ at $30^{\circ} \mathrm{C}$. A $0.5 \mathrm{~cm}^{3}$ aliquat of the mixture was transferred to the cuvette. Finally, a $0.50 \mathrm{~cm}^{3}$ portion of a $0.1 \mathrm{M}$ MOPS buffer solution (pH 7.0) containing HRP $\left(5.0 \times 10^{-8} \mathrm{M}\right)$ and FL (6.6× $10^{-4} \mathrm{M}$ ) was added to the mixture. The injection of the buffer solution initiated the $C L$ reaction immediately. Figure 1 (curve 1) shows a typical CL time profile. The CL intensity increased immediately after injection of the buffer solution, reached a maximum value in a few seconds, and then decreased. The CL emission ended within 2 min.

The simultaneous reaction sequence was investigated according to the procedure, except for using $1.0 \times 10^{-5} \mathrm{M}$ glucose. As can be seen in a typical CL time profile (curve 2) in Fig. 1, CL appears instantaneously upon injection of the $C L$ reagent solution and reaches a maximum within a few seconds, after which the intensity levels off for about $3 \mathrm{~min}$.

In Fig. 1 the maximum peak height for curve 2 is lower than that for curve 1; however, the CL emission in the simultaneous reaction sequence lasts for about $3 \mathrm{~min}$, which is longer than that in the two-stage reaction sequence. This implies that, in the simultaneous reaction sequence, hydrogen peroxide is successively generated during the glucose-GOD reaction, thus

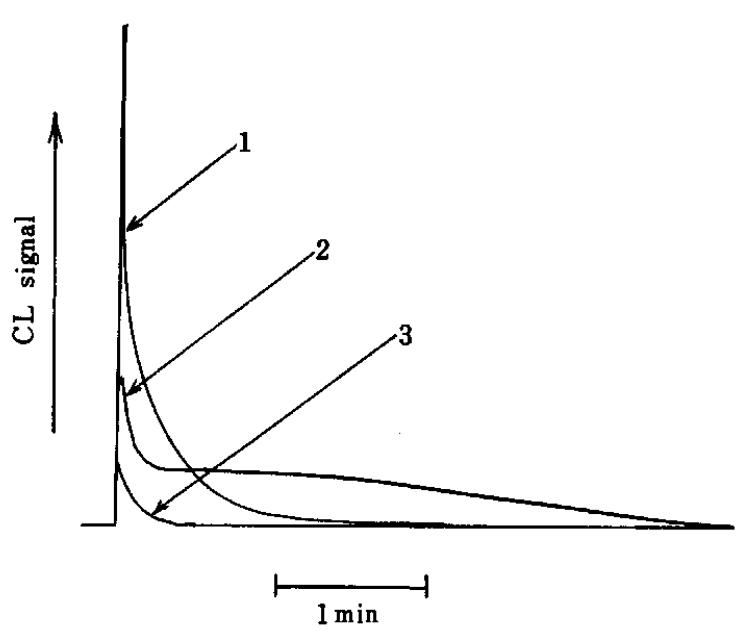

Fig. 1 Typical CL response curves. Curve 1: two-stage reaction sequence; 2 : simultaneous reaction sequence; 3 : blank signal.

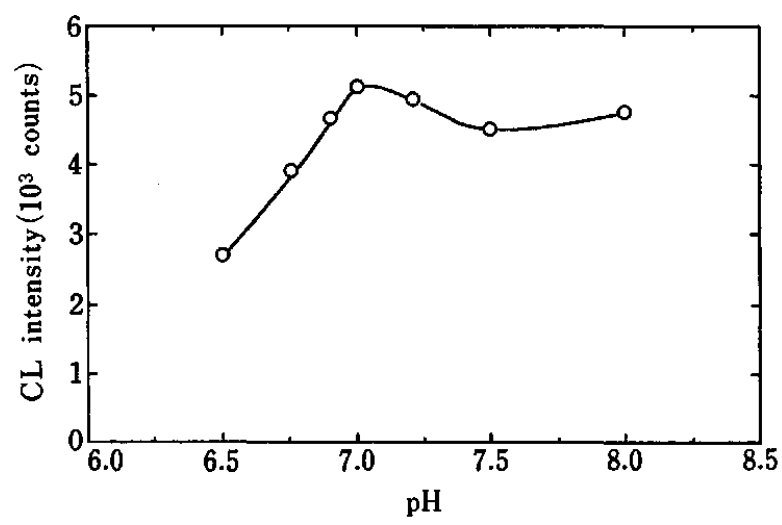

Fig. 2 Effect of $\mathrm{pH}$ on the $\mathrm{CL}$ intensity. [HRP] $=5.0 \times 10^{-8}$ $\mathrm{M} ; \quad[$ fluorescein $]=6.6 \times 10^{-4} \mathrm{M} ; \quad[$ glucose $]=5.0 \times 10^{-7} \mathrm{M}$; $[\mathrm{GOD}]=4.0 \mathrm{U} \mathrm{cm}^{-3}$.

emitting CL via the FL CL reaction.

The CL intensity for the two-stage reaction sequence was $2.2 \times 10^{4}$ counts, whereas it was $3.9 \times 10^{4}$ counts for the simultaneous reaction sequence when integrated for $3 \mathrm{~min}$ on a common sample of $1.0 \times 10^{-5} \mathrm{M}$ glucose. The latter method is thus more sensitive than the former. Moreover, in the latter method glucose can be simply determined by only mixing the CL reagent with a sample; the time required for a measurement of the CL emission is $3 \mathrm{~min}$. Accordingly, the simultaneous reaction sequence was adopted in the subsequent studies.

\section{Optimization of the reaction conditions}

We tried to determine the optimum conditions for a simultaneous coupling of the two reactions. The influence of the $\mathrm{pH}$ on the $\mathrm{CL}$ intensity was examined in the $\mathrm{pH}$ range from 6.5 to 8.0. As depicted in Fig. 2, the $\mathrm{CL}$ intensity was maximum at $\mathrm{pH}$ 7.0. A buffer of pH 7.0 was therefore chosen for the following studies.

The variation of the $C L$ intensities was examined as a 


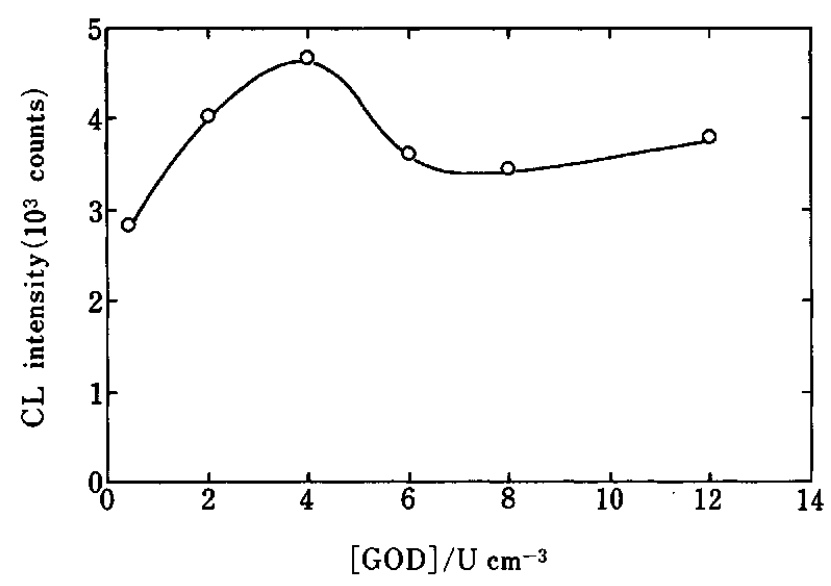

Fig. 3 Variation in the $\mathrm{CL}$ intensity with GOD concentration. $\left[\right.$ HRP] $=5.0 \times 10^{-8} \mathrm{M}$; [fluorescein] $=6.6 \times 10^{-4} \mathrm{M}$; [glucose] $=5.0 \times 10^{-7} \mathrm{M} ; \mathrm{H} 7.0$.

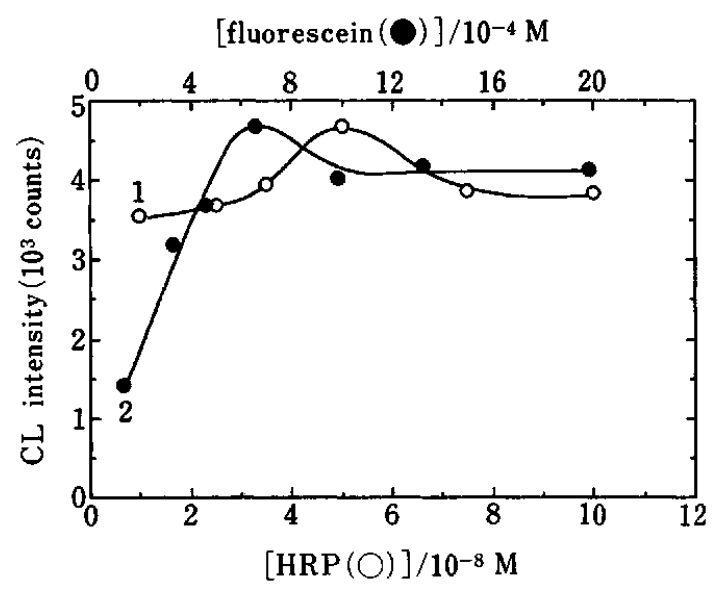

Fig. 4 Effect of the fluorescein and HRP concentrations on the CL intensity. [glucose] $=5.0 \times 10^{-7} \mathrm{M} ;[\mathrm{GOD}]=4.0 \mathrm{U} \mathrm{cm}^{-3}$; pH 7.0. Curve 1: HRP concentration was varied; [fluorescein] $=6.6 \times 10^{-4} \mathrm{M}$. Curve 2: fluorescein concentration was varied; $[$ HRP $]=5.0 \times 10^{-8} \mathrm{M}$.

function of the GOD concentration (Fig. 3). The CL intensity increased with an increase in the GOD concentration, and was maximum at $4.0 \mathrm{U} \mathrm{cm}^{-3}$ of GOD, above which the CL intensity decreased. A GOD concentration of $4.0 \mathrm{U} \mathrm{cm}^{-3}$ was thus selected for further studies.

Figure 4 illustrates the effect of the HRP (curve 1) and FL (curve 2) concentrations on the CL intensity. The $\mathrm{CL}$ intensity was maximum at $5.0 \times 10^{-8} \mathrm{M}$ HRP and $6.6 \times 10^{-4} \mathrm{M} \mathrm{FL}$, respectively. These concentrations were thus adopted in the recommended procedure.

Finally, the method of reagent mixing was examined. One method was to inject a mixed solution of GOD, HRP and FL into a glucose solution using a single flow line. The other was to inject two solutions (one was

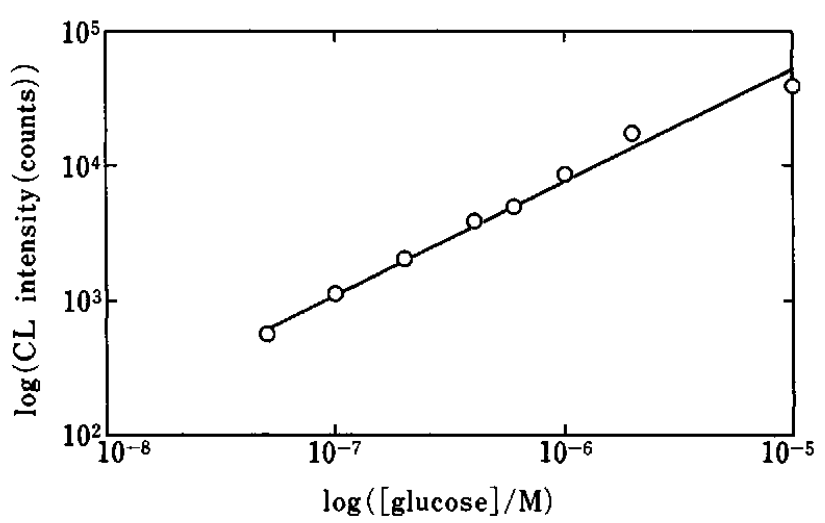

Fig. 5 Calibration curve for glucose. $[\mathrm{HRP}]=5.0 \times 10^{-8} \mathrm{M}$; [fluorescein] $=6.6 \times 10^{-4} \mathrm{M} ;[\mathrm{GOD}]=4.0 \mathrm{U} \mathrm{cm}^{-3} ; \mathrm{pH} 7.0$.

GOD, and the other a mixture of HRP and FL) into a glucose solution using two flow lines. As a result, the latter gave higher blank signals than did the former; the injection of a mixed solution of HRP, GOD, and FL to a glucose solution is thus recommended in the procedure. The injection of $0.5 \mathrm{~cm}^{3}$ of the mixed solution gave highly reproducible $\mathrm{CL}$ signals.

\section{Calibration curve for glucose}

Under the optimized conditions, thus determined, a calibration curve for glucose was prepared (Fig. 5). The $\log -\log$ plot of the CL intensity against the glucose concentrations is linear over the range from a detection limit of $5.0 \times 10^{-8} \mathrm{M}$ to $1.0 \times 10^{-5} \mathrm{M}$ glucose. The detection limit is defined as the glucose concentration yielding a CL intensity equal to twice the standard deviation of the blank CL intensity. The relative standard deviation of five successive determinations was $1.8 \%$ at a level of $5.0 \times 10^{-7} \mathrm{M}$. The detection limit by the present method was superior to that by a peroxyoxalate CL method using micelle $\left(6 \times 10^{-7} \mathrm{M}\right)^{9}$, and that by a luminol CL method using reversed micelle $\left(5.4 \times 10^{-6} \mathrm{M}\right) .^{5}$

The effects of diverse components, which are known to exist in serum, were examined on a synthetic sample at a concentration of $5.0 \times 10^{-7} \mathrm{M}$ glucose. The obtained data are listed in Table 1. The tolerance limit ratio is defined as the concentration ratio of the respective component against the glucose concentration giving an error less than $3 \%$. Uric acid, ascorbic acid, oxalic acid, lysine, tyrosine and most metal ions, except for manganese(II) ion, gave a negative error; cysteine, manganese(II) ion and nitrate ion caused a positive error.

\section{Determination of glucose in serum}

Two points must be considered when applying the present method to the determination of glucose in serum. One is its concentration level, which ranges from $4 \times 10^{-3}$ to $6 \times 10^{-3} \mathrm{M}$ in healthy subjects. The present method is therefore too sensitive to serum glucose. In view of this, the serum was diluted precisely 10000 -fold with water prior to the glucose assay. The other is the interference 
Table 1 Tolerance limit ratio

\begin{tabular}{lc}
\hline \multicolumn{1}{c}{ Compound } & Tolerance limit ratio \\
\hline $\mathrm{Cl}^{-}, \mathrm{K}^{+}, \mathrm{Na}^{+}, \mathrm{NH}_{4}^{+}, \mathrm{Mg}^{2+}, \mathrm{Ca}^{2+}$, & $<100$ \\
tryptophan, alanine, glutathione & \\
$\mathrm{NO}_{3}-$, lysine & 50 \\
$\mathrm{Oxalic}^{2+}$ acid & 10 \\
$\mathrm{Zn}^{2+}$, cysteine & 2 \\
$\mathrm{Mn}^{2+}$, tyrosine & 1 \\
$\mathrm{Al}^{3+}$ & 0.2 \\
$\mathrm{Fe}^{3+}$ & 0.1 \\
Uric acid & 0.08 \\
$\mathrm{Cu}^{2+}$, ascorbic acid & 0.02 \\
\hline
\end{tabular}

Glucose: $5 \times 10^{-7} \mathrm{M}$.

of diverse components present in serum. However, this can be neglected because the concentration ratios of such components to glucose in serum are well below the tolerance limit ratio given in Table 1.

Finally, two control serum samples were analyzed by the present method, and concomitantly by the colorimetric method with $o$-dianisidine as a chromogenic reagent. ${ }^{13}$ The results (in $\mathrm{mM}$ glucose) were, respectively, 4.32 and 4.33 for sample 1 , and 5.34 and 5.38 for sample 2. This clearly shows that glucose in serum can be determined by a simultaneous coupling of the GOD reaction with the FL CL reaction. A single determination takes only $3 \mathrm{~min}$. The present method is simple, highly sensitive and selective, and can be readily applied to routine analyses. The present FL CL reaction will be useful for quantifying any other substrates which are enzymatically converted into hydrogen peroxide.

\section{References}

1. A. Kunst, B. Draeger and J. Zeigenhorn, "Methods of Enzymatic Analysis", ed. U. Bergmeyer, J. Bergmeyer and M. Grassl, pp. $178-185$, Verlag Chemie, Weinheim, 1985.

2. A. Dahquest, Biochem. J., 80, 547 (1961).

3. D. T. Bostik and D. M. Hercules, Anal. Chem., 47, 447 (1975).

4. J. P. Auses, S. L. Cook and J. T. Maloy, Anal. Chem., 47, 244 (1975).

5. S. Igarashi and W. L. Hinze, Anal. Chem., 60, 446 (1988).

6. D. C. Williams III, G. F. Huff and W. R. Seitz, Anal. Chem., 48, 1003 (1976).

7. K. Nakashima, K. Maki, S. Kawaguchi, S. Akiyama, Y. Tsukamoto and K. Imai, Anal. Sci., 7, 709 (1991).

8. P. Zoonen, I. Herder, C. Gooijer, N. H. Velsorst, R. W. Frei, E. Kuntzberg and G. Gubitz, Anal. Lett., 19, 1949 (1986).

9. M. S. Abdel-Latif and G. G. Guilbault, Anal. Chem., 60, 2671 (1988).

10. K. Akimoto, Y. Shinmen, M. Sumida, S. Asami, T. Amachi, H. Yoshizumi, Y. Saeki, S. Shimizu and H. Yamada, Anal. Biochem., 189, 182 (1990).

11. T. Segawa, T. Kamidate and H. Watanabe, Anal. Sci., 6, 763 (1990).

12. T. Segawa, T. Kamidate and H. Watanabe, Nippon Kagaku Kaishi, 1992, 956 (1992).

13. I. Miura, J. Okada, K. Maeda and G. Okuda, Clin. Chim. Acta, 37, 538 (1972).

(Received July 22, 1992)

(Accepted August 20, 1992) 